\title{
Improving Real-Time Pedestrian Detectors with RGB+Depth Fusion
}

\author{
Tanguy Ophoff, Kristof Van Beeck, Toon Goedemé \\ EAVISE - Campus De Nayer, KU Leuven \\ J. De Nayerlaan 5, 2860 Sint-Katelijne-Waver, Belgium \\ firstname. lastname@kuleuven.be
}

\begin{abstract}
In this paper we investigate the benefit of using depth information on top of normal RGB for camera-based pedestrian detection. Indeed, depth sensing is easily acquired using depth cameras such as a Kinect or stereo setups. We investigate the best way to perform this sensor fusion with a special focus on lightweight single-pass CNN architectures, enabling real-time processing on limited hardware. We implement different network architectures, each fusing depth at different layers of our network. Our experiments show that midway fusion performs the best, outperforming a regular RGB detector substantially in accuracy. Moreover, we prove that our fusion network is better at detecting individuals in a crowd, by demonstrating that it has both a better localization of pedestrians and is better at handling occluded persons. The resulting network is computationally efficient and achieves real-time performance on both desktop and embedded GPUs.
\end{abstract}

\section{Introduction}

Fast and highly accurate pedestrian detection is a key ingredient for a manifold of applications in the safety and security domains. An example is the surveillance of public facilities such as airports or safety applications like pedestrian collision avoidance systems for autonomous vehicles. For the latter application the demands are very stringent, both w.r.t. detection accuracy as well as in its real-time behavior. The current state-of-the-art literature describes highly accurate detection systems. However, there are still several issues that remain to be solved. Detecting individual persons in a crowd, or even detecting highly occluded people are some of the problems current detection systems are facing. Besides providing highly accurate detections, a lot of applications require these detectors to process images in real-time. Important steps have been taken towards real-

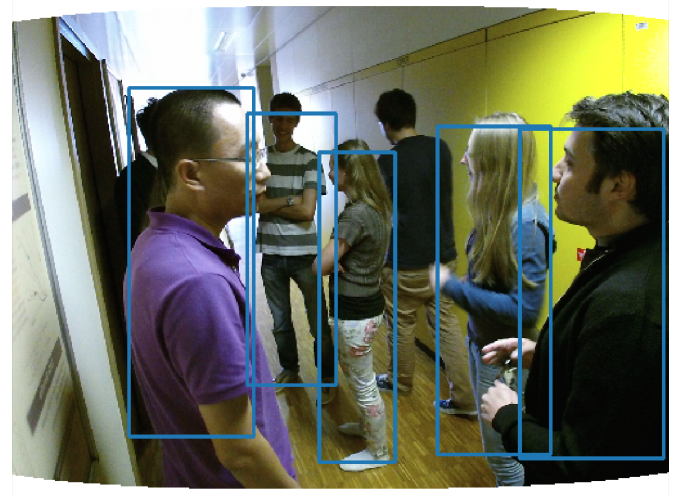

(a) Detections of the RGB detector

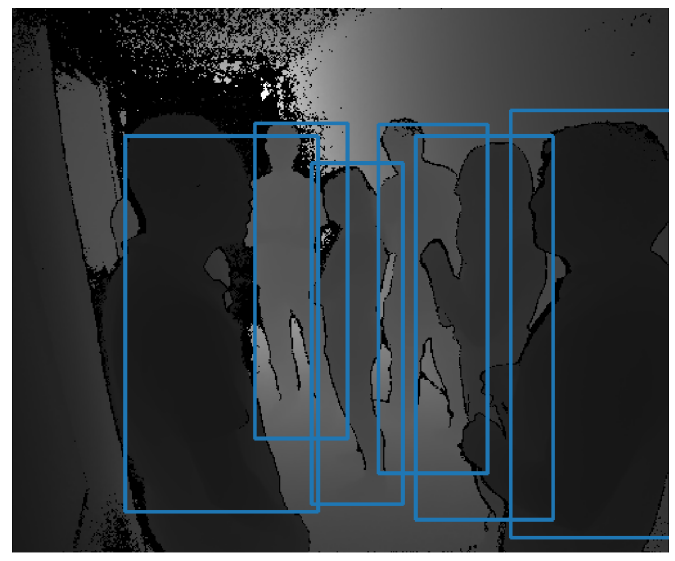

(b) Detections of the RGBD Mid Fusion detector, shown on the depth map

Figure 1: Example RGBD image pair. Note the easily distinguishable silhouettes of each person in the depth channel (b).The results of our RGB and RGBD detector are shown with bounding boxes. Our fusion network is able to detect all persons while having a better localization as compared to the RGB network.

978-1-5386-9294-3/18/\$31.00 (c)2018 IEEE 
time detection systems with single-pass convolutional neural networks $(\mathrm{CNN})$. A weakness these real-time detection networks have, is the difficulty to provide accurate bounding boxes around the detected pedestrians. This can be important for further segmentation or re-id applications.

Looking back at traditional computer vision techniques, we see that combining information from different types of sensors has been successfully used to increase the performance of detection systems. More recent, deep learning based techniques have also been proven to benefit from sensor fusion with e.g. thermal imaging.

In this paper we explore the added value of adding a depth channel to RGB images in a real-time pedestrian detection network. Commercial products like the Kinect or stereo based depth still remain a cheaper alternative than e.g. IR cameras for sensor fusion. Furthermore, depth is an interesting channel as it provides a more simple depiction of the environment with basic silhouettes of the objects. This allows a network to detect individuals in a crowd more easily, as each person has a different distance from the camera, resulting in clearly distinguishable silhouettes (see figure $1 b$ ). Another hypothesis we investigate, is that silhouettes in the depth channel can help to provide more accurate bounding boxes around the detected pedestrians.

Our main contributions are:

- We prove that depth is an interesting channel that adds valuable information for a detection network and increases the accuracy results compared to RGB detectors.

- We implemented a single-stage detection network with sensor fusion, capable of processing images in realtime.

- We relabeled an existing RGBD dataset, providing more information about the occlusion of people and made it publicly available. ${ }^{1}$

- We created an open-source library, re-implementing the YOLOv2 detector [20] in PyTorch and improving it with sensor fusion capabilities. ${ }^{2}$

In the remainder of this paper we will first discuss the state-of-the-art for pedestrian detection systems. We take a look at how sensor fusion improved classical computer vision techniques and how it has been recently used to improve deep neural networks for pedestrian detection. In section 3 we then explain how we improved the YOLOv2 detector [20] with sensor fusion capabilities and look at the different architectures we implemented. The achieved results are then discussed in section 4 , followed by our conclusion in section 5 .

\footnotetext{
${ }^{1}$ www.eavise.be/dataset-rgbd-pedestrian-relabeled

${ }^{2}$ https://www.gitlab.com/eavise/lightnet
}

\section{Related Work}

Traditional computer vision techniques used handcrafted features as input to a learned classifier to perform various tasks such as object and pedestrian detection $[4,5,6]$. In order to increase the accuracy, these techniques have been successfully extended by using features from various different sources like thermal imaging [3,12] and depth [13,23]. Benenson et al. [2] also proved that depth can be a strong queue for preprocessing images, reducing the detection search space.

However, with the rise of CNNs, deep learning has been outperforming traditional computer vision techniques by a significant margin $[11,15]$. Pedestrian detection - or object detection in general - is often based on a combination of a CNN for classification with a sliding-window approach $[9,24]$. While these techniques have proven to be successful, a major drawback is that they are very computationally expensive. These techniques generate a great number of region proposals, of which each need to be evaluated by a classification network. These issues have been partially solved by sharing computations between the region proposal and classification pipelines [8,21].

Finally, the current state-of-the-art for fast object detection lies with single-pass detection networks, such as YOLO [19,20] and SSD [18]. By re-framing object detection as a single regression problem, one can use a single network which simultaneously outputs bounding box coordinates and class scores. Beside the fact that these networks are orders of magnitude faster than previous work, they also have the advantage of considering the entire image when computing bounding boxes. This additional information makes for even better detectors, outperforming previous detection pipelines.

While these state-of-the-art detection networks have achieved impressive results for pedestrian detection, there are still several issues that remain to be solved. Taking inspiration from traditional computer vision techniques, some of these issues have been solved by combining these detectors with sensor fusion $[14,17,25,26]$. For example, adding extra information in the form of thermal imaging allowed these detectors to perform better in challenging day-andnight scenarios [25]. Zhou et al. [27] took initial steps to fuse depth information with CNNs. However, their approach still consists of a suboptimal two-stage approach. To the best of our knowledge, depth has never been fused in a single-pass detector.

Our work thus significantly diverges from existing approaches. We fuse depth in a single-pass detector to improve detection results of individual persons in a crowd, as this remains one of the main weaknesses of single-pass detectors. 


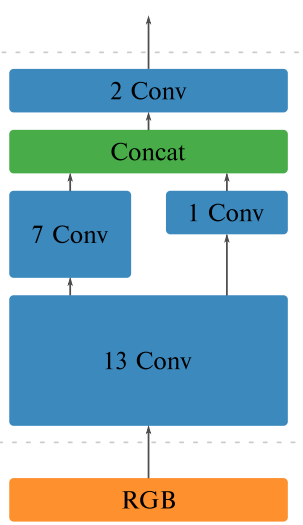

(a) YOLOv2

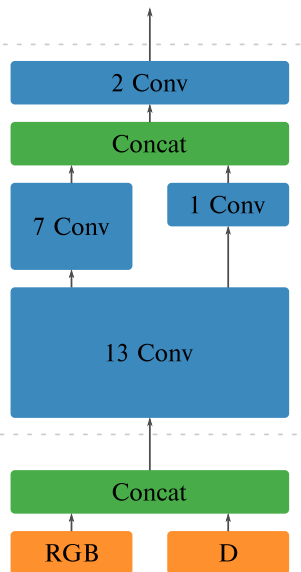

(b) YOLOv2 with Early Fusion

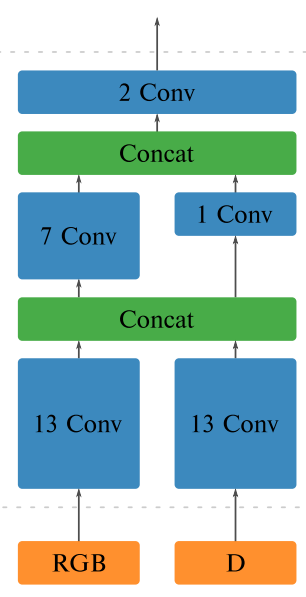

(c) YOLOv2 with Mid Fusion

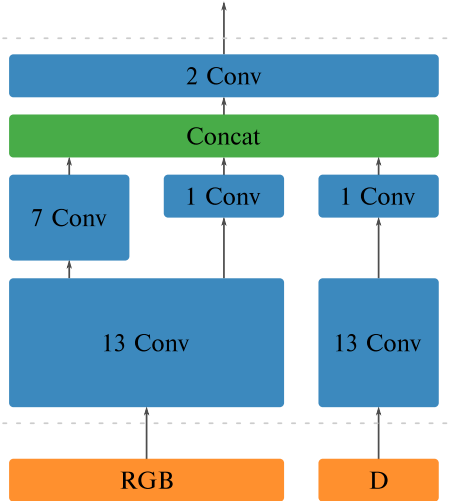

(d) YOLOv2 with Late Fusion

Figure 2: Different fusion architectures that were implemented and compared with each other.

\section{Fusion Network Architectures}

We assume the input of our algorithm consists of a sensor that both acquires an RGB image, as well as a depth image (D). Both are assumed to be perfectly aligned with each other, yielding a 4-channel RGBD image. The latter can easily be realized with an RGBD range camera based on structured light, time-of-flight or stereo.

Below, we investigate the influence of different network architectures for RGBD fusion, as depicted in figure 2. Fusing information at different layers of a network is possible, as inspired by work on IR fusion [17,26]. We start from the YOLOv2 architecture, a state-of-the-art single-pass detection network consisting of 23 convolutional layers [20] and initially fuse depth at the first convolutional layer, coined Early Fusion (see section 3.1). YOLOv2 has a pass-through layer, concatenating high resolution features from an early convolution with lower resolution features later in the network. Subsequently, we explore the fusing of depth before (Midway Fusion) and after (Late Fusion) this pass-through layer (see section 3.2).

Our different networks are all trained and evaluated on the EPFL RGBD Pedestrian Dataset [1], which provides highly accurate depth maps, captured with a Kinect V2 and calibrated to align with the RGB images. However, the original labeling of this dataset omits bounding boxes for highly occluded persons. Thus we decided to relabel the entire datase $^{1}$ manually, annotating every person - independent of their occlusion level - and instead adding an extra occlusion percentage (in steps of $25 \%$ ) that provides information about how much a person is occluded in the image. These extra labels were then used during the analysis of our networks (see section 4.3). Because the Kinect only has a limited range, we also set a lost flag for persons that fell outside the range of the Kinect sensor and thus were only visible in the RGB camera. During both training and evaluation, these lost annotations are then ignored, meaning it does not matter for the metric whether these persons are detected. This is indeed the fairest when evaluating the added value of depth for pedestrian detection.

\subsection{Early Fusion}

The simplest form of fusion one can implement, is adding the sensor data as an extra input channel. This means that only the first convolutional layer of the early fusion network is different than the default RGB detector (see figure $2 b$ ). We initialized the weights of this network with pretrained weights from the darknet19 classification network [20], which was trained on ImageNet. The extra D weights from the first layer were randomly initialized using a normal Kaiming initialization [10], as it improves convergence rates for deep neural networks with rectifying linear units (ReLU).

\subsection{Mid and Late Fusion}

Liu et al. [17] prove that fusing multispectral data at a later stage in a neural network is beneficial for the detection network, hinting that the same holds for fusing depth information in a network. Thus we implement two different networks, fusing depth features right before and after the pass-through layer of our detector (see figures $2 c$ and $2 d$ ). For the late fusion network, we tried two different variations with $13+7$ and $13+1$ convolutional layers for the depth subnetwork. As both networks performed equally well, we will only discuss the $13+1$ variant which has fewer computations. The weights of the RGB subnetwork were initialized with pretrained weights from the darknet19 classifica- 
tion network [20], whilst the weights of the depth subnetwork were initialized from a depth-only network, which we trained on our relabeled EPFL dataset. Finally, the weights of the layers after the concatenation of both subnetworks were randomly initialized, again using a normal Kaiming initialization scheme [10].

\section{Experiments and Results}

We trained and evaluated the different networks on our relabeled EPFL RGBD Pedestrian Dataset [1]. For this purpose the dataset was split in a train, validation and test set according to a 70-10-20\% division. As is customary with video sequences, every sequence of the dataset was individually split in a sequential manner and the resulting split sequences were then combined to form the different sets $[7,22]$. The rationale behind this method is that consecutive frames of a video sequence do not differ a lot. Having adjacent - and thus similar - frames of a video all in the same subset will cause a network, that is overfitting on the training set, to perform worse on the validation and test set.

All networks were trained for 10000 batches of 64 images, with tests running every 200 batches on the validation set. The same data augmentation techniques were used as for YOLOv2 in the darknet framework [20]. Finally, we evaluated the best performing network on the validation set on our test set. Besides training the different fusion networks, we also trained and evaluated a standard YOLOv2 network on the RGB images and on the depth maps. This provides a baseline result to compare, as well as insight in the value of the depth maps for detection.

We performed extensive experiments, which demonstrate that depth fusion improves detection networks by increasing the accuracy, providing better localization and more easily detecting occluded persons, as explained below.

\subsection{Accuracy}

Figure 3 shows the Precision-recall curves (PR-curves) of our different networks. These results were generated with an intersection over union (IoU) threshold of $50 \%$. First and foremost, we can see that a default YOLOv2 network achieves better results with only depth maps than with RGB. The fusion networks manage to use this extra information and outperform both the RGB and depth networks. As is the case with IR [17], mid fusion gives the best results, outperforming the RGB detector with almost $3 \%$ average precision (AP).

However, when we consider the early fusion network, we can see that it falls short of the two other fusion networks. We believe this is due to the strong bias, when initializing the weights of the network with pretrained weights from a pure RGB network.

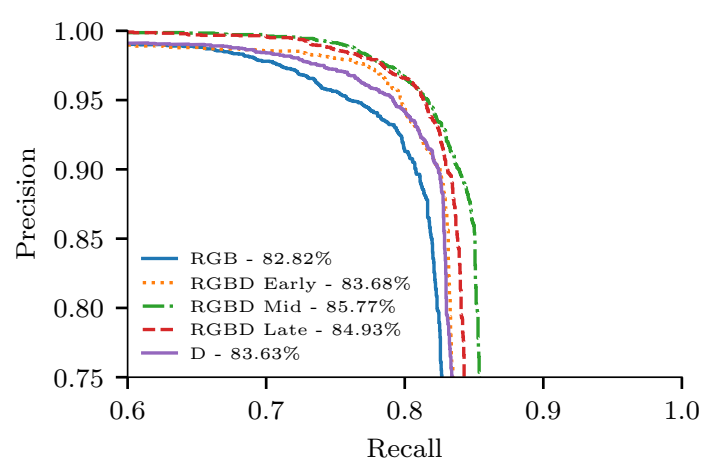

Figure 3: PR-curves and AP scores of the different networks on our relabeled dataset. Detections with $I o U \geq 50 \%$ were counted as true positive. Note that the graph is zoomed in for clarity.

\subsection{Localization}

Another advantage of including depth information in a network, can be found in the fact that the clearly distinguishable silhouettes in the depth maps allow fusion networks to provide more accurate bounding boxes around the detected pedestrians (see figure $1 b$ ). To evaluate this, we measure the AP of our networks, using the new COCO IoU thresholding scheme [16]. This method measures the AP of a network at increasing IoU scores, going from $50 \%$ to $95 \%$ with steps of $5 \%$, and then averages all these scores to compute a single value. This metric rewards techniques that offer a better localization and is thus a good fit for proving our hypothesis.

As can be derived from Table 1, the fusion networks perform much better than the RGB network, with mid fusion being the best with an increase of $13.5 \%$ AP. Moreover, the increase in AP is more prominent when using this new COCO metric, which is a clear indication that the fusion networks have a better localization than the RGB network, proving our hypothesis.

It can again be noted that the early fusion network performs suboptimal, achieving worse results than a depthonly network. This further pushes our belief that the pretrained RGB weights cause a strong bias, making the network ignore the extra depth information.

\subsection{Occlusion}

A second hypothesis we want to demonstrate, is that depth helps with the detection of (partly) occluded persons. Single-pass detection networks are often unable to detect two or more individuals, when they are partially occluding each other. However, both persons will have a different distance from the camera, allowing our fusion networks to use the depth information to differentiate between them. In order to prove this, we measure the performance of our networks at different occlusion intervals. Figure 5 shows 


\begin{tabular}{|l|cc|ccc|}
\hline Architecture & $\mathbf{A P}_{\mathbf{5 0 \%}}$ & AP $_{\text {COCO }}$ & Mflops & FPS $_{\text {GTX1080Ti }}$ & FPS $_{\text {JetsonTX2 }}$ \\
\hline RGB & $82.82 \%$ & $41.23 \%$ & 29360 & 129.9 & 9.7 \\
D & $83.63 \%$ & $49.36 \%$ & 29161 & 133.7 & 10.2 \\
RGBD Early & $83.68 \%$ & $46.29 \%$ & 29460 & 128.7 & 9.6 \\
RGBD Mid & $85.77 \%$ & $54.74 \%$ & 44722 & 96.6 & 6.0 \\
RGBD Late & $84.93 \%$ & $53.33 \%$ & 45564 & 94.7 & 5.4 \\
\hline
\end{tabular}

Table 1: AP scores and speed of the different network architectures on images of $416 \times 416$. FPS was measured one image at a time on the test set and then averaged.

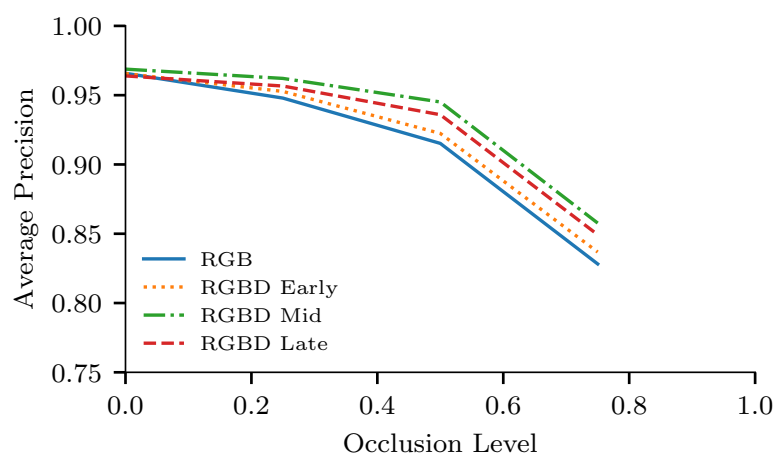

Figure 4: AP scores of our different networks at increasing occlusion levels. Note that the AP of our RGB network drops faster than the scores of our fusion networks.

the different PR-curves of our networks, which were computed whilst ignoring people that were occluded more than the marked interval. Figure 4 plots the average precision of each.

When only regarding non-occluded persons, all networks perform comparatively well (see figure 5a). However, when we start including people with higher occlusion levels, the AP of the RGB network deteriorates faster than the AP of our fusion networks (see figure 4). This proves that our fusion networks are more capable of detecting occluded persons in our dataset.

\subsection{Speed}

One might argue that, due to the increased amount of convolution layers, our fusion networks are slightly more computationally expensive. In Table 1 we list the number of floating point operations for one image of size $416 \times 416$. The mid and late fusion networks require around 1.5 times more operations per image. While this indeed means the networks perform slower, they still reach almost 100 FPS on a commercial GPU (Nvidia GTX $1080 \mathrm{Ti}$ ), satisfying our real-time constraint. This is fast enough to $e$.g. process multiple surveillance camera streams simultaneously on a normal desktop PC. Even on embedded devices (Nvidia Jetson TX2) our PyTorch pipeline reaches more than 5 FPS, which is fast enough for a great number of applications.

\section{Conclusion and Future Work}

In this paper we proposed the fusion of RGB and depth images using single-pass detection networks, capable of processing images in real-time. We evaluated different network architectures, fusing the depth information at different layers of our network and determined that midway fusion performs the best, with an increase of almost $3 \%$ AP on our relabeled EPFL RGBD Pedestrian dataset.

Moreover, the use of depth information allows our fusion network to detect individuals in a crowd more easily. We proved this by demonstrating that our fusion networks allow for a better localization of the pedestrians and perform better with occluded persons. Indeed, our midway fusion has an increase of $13.5 \%$ AP, when using the new COCO IoU thresholding scheme, clearly demonstrating a better localization. When increasing the allowed occluded percentage of the pedestrians, we noted that the RGB network deteriorates faster than our fusion networks.

Note that the main focus of this paper is to investigate the possible increase in accuracy depth fusion offers. Thus, evidently, the resulting networks can be further optimized for speed by concatenating layers or by quantizing the weights using frameworks like TensorRT.

In the future, we aim to investigate the influence of the quality of the depth maps on our fusion networks. The noisy depth maps used, acquired from a Kinect, could explain the relatively small increase in accuracy we achieve with our networks. In the future, we will investigate whether better depth acquisition might lead to an even more robust detection.

\section{Acknowledgements}

This work is partially supported by the FWO (SBO Project Omnidrone) and VLAIO.

\section{References}

[1] T. Bagautdinov, F. Fleuret, and P. Fua. Probability occupancy maps for occluded depth images. 2015.

[2] R. Benenson, M. Mathias, R. Timofte, and L. Van Gool. Pedestrian detection at 100 frames per second. In $C V P R$, pages 2903-2910. IEEE, 2012. 


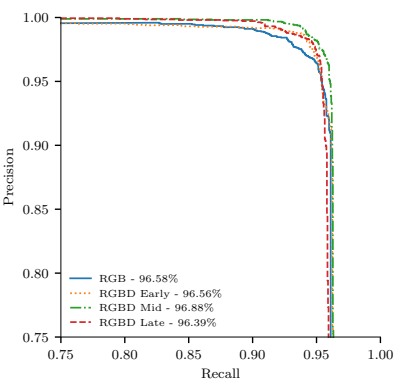

(a) $0 \%$ occluded

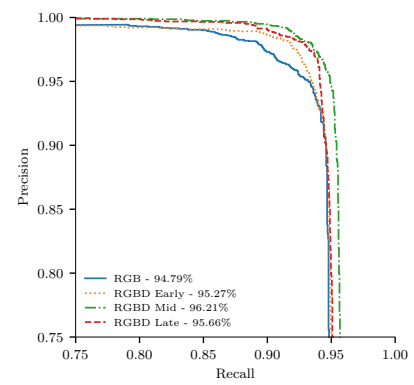

(b) $0-25 \%$ occluded

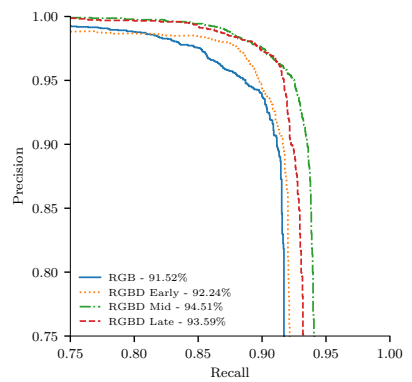

(c) $0-50 \%$ occluded

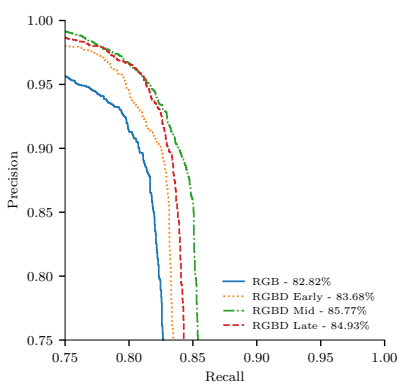

(d) $0-75 \%$ occluded

Figure 5: PR-curves and AP scores of the different networks with increasing occlusion levels. Note that the graphs are zoomed in for clarity.

[3] F. De Smedt, S. Puttemans, and T. Goedemé. How to reach top accuracy for a visual pedestrian warning system from a car? In 2016 Sixth International Conference on Image Processing Theory, Tools and Applications (IPTA), pages 1-6, Dec 2016.

[4] P. Dollár, R. Appel, S. Belongie, and P. Perona. Fast feature pyramids for object detection. IEEE TPAMI, 36(8):15321545, 2014.

[5] P. Dollár, Z. Tu, P. Perona, and S. Belongie. Integral channel features. 2009.

[6] P. Felzenszwalb, D. McAllester, and D. Ramanan. A discriminatively trained, multiscale, deformable part model. In CVPR, pages 1-8, 2008.

[7] A. Geiger, P. Lenz, and R. Urtasun. Are we ready for autonomous driving? the KITTI vision benchmark suite. In CVPR, 2012.

[8] R. Girshick. Fast R-CNN. In ICCV, pages 1440-1448, Dec 2015.

[9] R. Girshick, J. Donahue, T. Darrell, and J. Malik. Rich feature hierarchies for accurate object detection and semantic segmentation. In CVPR, pages 580-587, 2014.

[10] K. He, X. Zhang, S. Ren, and J. Sun. Delving deep into rectifiers: Surpassing human-level performance on imagenet classification. In CVPR, pages 1026-1034, 2015.

[11] K. He, X. Zhang, S. Ren, and J. Sun. Deep residual learning for image recognition. In CVPR, pages 770-778, 2016.

[12] S. Hwang, J. Park, N. Kim, Y. Choi, and I. S. Kweon. Multispectral pedestrian detection: Benchmark dataset and baseline. Integrated Comput.-Aided Eng, 20:347-360, 2013.

[13] O. H. Jafari, D. Mitzel, and B. Leibe. Real-time RGB-D based people detection and tracking for mobile robots and head-worn cameras. In Robotics and Automation (ICRA), 2014 IEEE International Conference on, pages 5636-5643. IEEE, 2014.

[14] D. König, M. Adam, C. Jarvers, G. Layher, H. Neumann, and M. Teutsch. Fully convolutional region proposal networks for multispectral person detection. In CVPR Workshops, pages 243-250. IEEE, 2017.

[15] A. Krizhevsky, I. Sutskever, and G. E. Hinton. Imagenet classification with deep convolutional neural networks. In NIPS, pages 1097-1105, 2012.
[16] T.-Y. Lin, M. Maire, S. Belongie, J. Hays, P. Perona, D. Ramanan, P. Dollár, and C. L. Zitnick. Microsoft COCO: Common objects in context. In ECCV, pages 740-755. Springer, 2014.

[17] J. Liu, S. Zhang, S. Wang, and D. Metaxas. Multispectral deep neural networks for pedestrian detection. In $B M V C$, pages 73.1-73.13, September 2016.

[18] W. Liu, D. Anguelov, D. Erhan, C. Szegedy, S. Reed, C.-Y. $\mathrm{Fu}$, and A. C. Berg. SSD: Single shot multibox detector. In ECCV, pages 21-37. Springer, 2016.

[19] J. Redmon, S. Divvala, R. Girshick, and A. Farhadi. You only look once: Unified, real-time object detection. In CVPR, pages 779-788, 2016.

[20] J. Redmon and A. Farhadi. Yolo9000: Better, faster, stronger. In CVPR, pages 6517-6525, July 2017.

[21] S. Ren, K. He, R. Girshick, and J. Sun. Faster R-CNN: Towards real-time object detection with region proposal networks. In NIPS, pages 91-99, 2015.

[22] E. Ristani, F. Solera, R. Zou, R. Cucchiara, and C. Tomasi. Performance measures and a data set for multi-target, multicamera tracking. In European Conference on Computer Vision workshop on Benchmarking Multi-Target Tracking, 2016.

[23] L. Spinello and K. O. Arras. People detection in RGB-D data. In Intelligent Robots and Systems (IROS), pages 38383843. IEEE, 2011.

[24] C. Szegedy, A. Toshev, and D. Erhan. Deep neural networks for object detection. In NIPS, pages 2553-2561, 2013.

[25] M. Vandersteegen, K. Van Beeck, and T. Goedemé. Realtime multispectral pedestrian detection with a single-pass deep neural network. In Image Analysis and Recognition: 15th International Conference (ICIAR), 2018.

[26] J. Wagner, V. Fischer, M. Herman, and S. Behnke. Multispectral pedestrian detection using deep fusion convolutional neural networks. In 24th European Symposium on Artificial Neural Networks, Computational Intelligence and Machine Learning (ESANN), pages 509-514, 2016.

[27] K. Zhou, A. Paiement, and M. Mirmehdi. Detecting humans in RGB-D data with CNNs. In 2017 Fifteenth IAPR International Conference on Machine Vision Applications (MVA), pages 306-309, May 2017. 